\title{
Una revisión de la literatura experimental sobre los efectos motivacionales del alcohol y su modulación por factores biológicos y ambientales
}

\author{
María L. de la Torre*, María D. Escarabajal y Ángeles Agüero
}

Departamento de Psicología. Área de psicobiología. Universidad de Jaén

\begin{abstract}
Resumen: El alcohol es posiblemente la droga psicoactiva más consumida en el mundo. La ingesta moderada es común, pero para algunos individuos el consumo de alcohol se convierte en un trastorno adictivo. La progresión desde un consumo moderado de alcohol hasta el abuso podría deberse a la existencia de una sensibilidad diferencial, genéticamente determinada, a los efectos reforzantes y aversivos del alcohol. Así mismo, se sugiere que factores ambientales, como el estrés, podrían intervenir en el inicio del alcoholismo, influyendo sobre dicha sensibilidad diferencial y, en última instancia, sobre el consumo de alcohol. En el presente trabajo se revisa la literatura experimental existente acerca de los elementos protagonistas en la relación entre estrés y etanol. Por una parte se revisan los principales modelos de estrés existentes y, por otra, se aborda el estudio de las propiedades reforzantes y aversivas de esta droga, incluyendo datos sobre la influencia de variables como la edad, el sexo, y la experiencia previa con la droga. Finalmente, se expone un resumen de los datos existentes sobre la investigación con animales sobre la influencia del estrés en la percepción de las propiedades motivacionales opuestas del etanol. Nuestro objetivo es contribuir a clarificar el conocimiento existente acerca de las relaciones entre determinados factores biológicos (como el sexo o la edad) y ambientales (experiencia previa y estrés), y la conducta de ingesta de alcohol, poniendo de manifiesto la importancia de algunos factores de riesgo para el desarrollo del alcoholismo.

Palabras clave: Alcohol; estrés; refuerzo; aversión; adicción.
\end{abstract}

\section{Introducción}

El alcohol es posiblemente la droga psicoactiva más consumida en el mundo. La ingesta moderada es común, pero para algunos individuos el consumo de alcohol se convierte en un trastorno adictivo. Tradicionalmente se ha considerado a los factores genéticos como los principales determinantes del alcoholismo. La existencia de variaciones genéticas en la sensibilidad a los efectos del etanol es un hecho bien contrastado en roedores (Broadbent, Muccino y Cunningham, 2002, y Pautassi, Camarini, Quadros, Miczek, e Israel, 2010) y en humanos (Duranceaux et al., 2006; ver Escarabajal, 2003 para revisión). Pero en las últimas dos décadas dicha primacía ha sido de alguna manera moderada por la investigación epidemiológica y preclínica, que pone de manifiesto la importancia de factores relacionados con la experiencia.

Entre los factores relacionados con la experiencia, o factores ambientales, hemos de destacar el estrés. La investigación clínica indica que el estrés, no sólo es un factor de riesgo en el desarrollo de una adicción, sino también un disparador de las recaídas (ver, por ejemplo, Briand y Blendy, 2010; McDevitt-Murphy et al., 2010; Sakusic et al., 2010; Shina, 2005). Existe, además, un amplio conjunto de datos

* Dirección para correspondencia [Correspondence address]: $\mathrm{M}^{\mathrm{a}}$ Lourdes de la Torre Vacas. Departamento de Psicología. Área de psicobiología. Universidad de Jaén. Paraje Las Lagunillas, s/n 23071, Jaén (España). E-mail: mltorre@ujaen.es
Title: A review of experimental literature on alcohol's motivational effects and its modulation by biological and environmental factors.

Abstract: Alcohol is probably the most consumed psychoactive drug in the world. Controlled consumption of alcohol is common, but for many individuals this consumption becomes an addiction. The progression from an habitual consumption of alcohol to abuse of the substance could be due to the existence of a differential sensitivity, which would be genetically determined, to the reinforcing and aversive effects of alcohol. Likewise, it has been suggested that environmental factors such as stress might be involved in the onset of alcoholism, influencing on such a differential sensitivity and, ultimately, on the consumption of alcohol. In this paper it is reviewed the experimental published data on each of the elements of the relationship between stress and ethanol. Thus, on the one hand they are reviewed the main animals models of stress and, on the other hand, we approached the study of the reinforcing and aversive properties of ethanol, including data on the influence of variables such as age, sex, and previous experience with the drug. And, finally, we present a resume of data collected from animal research on the influence of stress on the perception of the motivational opposing properties of ethanol. Our purpose is help to clarify the existing knowledge about the relations between biological factors (such as sex or age) and environmental factors (previous experience and stress), and the behavior of alcohol intake, thus highlighting the importance of some factors of risk for developing alcoholism.

Key words: alcohol; stress; reinforcement; aversion; addiction.

procedentes de la investigación con animales que avalan la relación entre el estrés y los efectos de las drogas de abuso. Este tipo de estudios utiliza modelos animales que permiten estudiar las interacciones entre ambos factores (ver Lu, Shepard, Hall y Shaham, 2003; See y Waters, 2011). Se ha observado, por ejemplo, que las respuestas conductuales a cocaína, anfetamina y morfina, como actividad locomotora y estereotipias motoras, son aumentadas por el estrés. Por otra parte, el estrés potencia los niveles de dopamina (DA) inducidos por cocaína, y el aumento de la liberación de DA por parte del estriado, en respuesta a la anfetamina. Junto con esta sensibilización de la respuesta conductual y neuroquímica ante una droga, la exposición al estrés también facilita la auto-administración de sustancias psicoactivas, y el condicionamiento de preferencia por un lugar inducido por drogas (revisado en Briand y Blendy, 2010; Lu et al., 2003). Los datos concernientes a la relación específica entre estrés y los efectos motivacionales del etanol van en la misma línea, y serán revisados en un apartado posterior.

Los efectos de factores ambientales como el estrés sobre la propensión al abuso de sustancias estimulantes han sido razonablemente atribuidos a alteraciones en el sistema encefálico dopaminérgico mesolímbico, sistema crucial en las propiedades activadoras motoras y de refuerzo de las drogas estimulantes (ver Melis, Diana, Enrico, Marinelli y Brodie, 2009; Pani, Porcella y Gessa, 2000; Yap y Miczek, 2008, para revisión sobre el tema). Sin embargo, hemos de tener en cuenta que las drogas de abuso son reforzadores únicos que 
cuentan con la capacidad de generar tanto efectos reforzantes como aversivos, habiéndose sugerido que la vulnerabilidad a las drogas de abuso no está sólo determinada por la sensibilidad al refuerzo, sino que puede ser el resultado del balance entre los efectos motivacionales opuestos producidos por la droga (revisado en Roma, Davis, Kohut, Huntsberry y Riley, 2008). De hecho, las consecuencias apetitivas y aversivas del etanol incrementan y decrecen, respectivamente, la probabilidad subsecuente de las conductas de búsqueda y consumo de alcohol (revisado en Pautassi, Nizhnikov y Spear, 2009; Wilson, Burghardt, Ford, Wilkinson y Primeaux, 2004; ver también Cunningham, Clemans y Fidler, 2002; Broadbent, Muccino y Cunningham, 2002; Green y Grahame, 2008). El estudio de estos factores motivacionales parece ser particularmente importante para la comprensión de por qué ciertos individuos progresan rápidamente desde un uso controlado del alcohol, al abuso y la dependencia, mientras que otros continúan con el consumo controlado a pesar de la continua exposición a la droga.

Teniendo en cuenta estos antecedentes, y otros datos publicados (Roma, Rinker et al., 2008), cabe pensar que se dé una interacción entre factores genéticos y ambientales, como el estrés, para modular la sensibilidad diferencial a los efectos contrapuestos, reforzantes y aversivos, del alcohol, influenciando de esta forma la conducta de ingesta.

A continuación se revisa la literatura experimental existente acerca de los elementos protagonistas en la relación entre estrés y etanol. Por una parte se revisa el estudio experimental del estrés, y, por otra se aborda el estudio de las propiedades reforzantes y aversivas del etanol, incluyendo datos sobre la influencia de variables como la edad, el sexo, y la experiencia previa con la droga. Y, finalmente, se expone un compendio de datos existentes sobre la influencia del estrés en la percepción de las propiedades motivacionales opuestas del etanol.

\section{E1 estrés}

Los estímulos y respuestas de estrés son muy inespecíficos, lo que implica que el número de modelos animales para su estudio experimental sea variado y elevado dando lugar a una clasificación.

En todos los modelos de estrés se sitúa al sujeto experimental en condiciones potencialmente nocivas, pudiendo clasificarse estos estímulos en dos grupos: algógenos y no algógenos (Alonso y Navarro, 1993; Steckler, 2005). Entre los primeros, el más utilizado es la estimulación eléctrica de la superficie de apoyo. Sin embargo, conviene resaltar que el estrés producido por dolor es difícil de interpretar, dado que con frecuencia no es posible diferenciar entre los efectos inducidos por el dolor y los propios del estrés (Alonso y Navarro, 1993). Los estímulos no algógenos son de diversa naturaleza, pero en la mayor parte de los casos consisten en manipulaciones del componente social (aislamiento, separación maternal, derrota social, etc.).
Qué estresores conforman exactamente la categoría de estresores algógenos (o físicos), y cuáles la de estresores no algógenos (o psicológicos) es, en ocasiones, un tema de debate. Cada uno de ellos parece activar diferentes circuitos neuronales. Los circuitos límbicos que conectan el hipocampo, complejo amigdalino y corteza prefrontal son sensibles a estresores eminentemente psicológicos. Por el contrario, las amenazas de naturaleza física activan las vías eferentes viscerales y somatosensoriales que se dirigen directamente al núcleo paraventricular (NPV) del hipotálamo (parte del eje hipotálamo-hipófisis-adrenal, HHA). En este caso, la rápida activación, de la base del encéfalo y del hipotálamo, evita el procesamiento cognitivo en las áreas límbicas y corticales. Según esta distinción entre estresores "límbicos" y "no límbicos", Herman y Cullinan (1997) propusieron dos vías generalizadas de estrés, la sistémica y la procesadora. De acuerdo con su hipótesis, los estímulos respiratorios, cardiovasculares o inmunes, representan estresores sistémicos, que requieren reacciones inmediatas para la supervivencia pero no una interpretación de estructuras cerebrales de orden superior. Por el contrario, los estímulos psicológicos, o de procesamiento, necesitan del procesamiento cortical y, dependiendo de la experiencia previa, la información es integrada en las estructuras límbicas, para después inducir las respuestas neuroendocrinas y conductuales (revisado en Fuchs y Flügge, 2003).

Para la inducción de estrés, la mayor parte de los estudios con animales de laboratorio han usado descargas eléctricas aplicadas en cola o patas, e inmovilización por restricción física y natación forzada. La característica más atractiva de estos estresores es el control directo de todos los parámetros relevantes (intensidad, duración, frecuencia y controlabilidad), reduciendo así, en gran medida, la variabilidad de experiencias entre los sujetos (véase Gambrana, 2007). Sin embargo, la necesidad de obtener mayor validez ecológica ha llevado a utilizar estresores como la separación maternal, la privación de alimento y la derrota social, presentados de forma intermitente y continua (Miczek, Yap, y Covington, 2008). La separación maternal involuntaria repetida es un estrés vital temprano que puede disparar una cascada de eventos neurobiológicos que persisten en la edad adulta (Roma, Davis et al., 2008). La privación aguda de alimento puede mimetizar las condiciones naturales de un periodo moderado de escasez de alimento (Gambrana, 2007). Tanto para roedores como para humanos, el estatus social es importante, y las derrotas sociales repetidas y la intimidación pueden inducir cambios fisiológicos y conductuales, a corto y a largo plazo (Miczek et al., 2008; Yap y Miczek, 2008). Estos estresores intermitentes inducen secuelas conductuales y neurobiológicas que parecen afectar directamente al proceso de adicción a una droga.

Otros estresores son de naturaleza farmacológica. Y, aunque estos tienen limitaciones, particularmente el hecho de no modelar completamente el impacto de un estresor externo, desde una perspectiva experimental el estrés inducido farmacológicamente ofrece una clara ventaja al actuar a 
través de mecanismos neurobiológicos similares en humanos y en animales de laboratorio. Además, el uso de estresores farmacológicos proporciona un medio de valorar la activación del estrés de una forma dosis-dependiente que es difícil valorar mediante estresores externos. See y Waters (2011) han revisado los estudios publicados que han utilizado agentes farmacológicos como inductores de estrés vinculados con la conducta de búsqueda de drogas subsecuente y concluyen que la inducción farmacológica de estados de estrés ofrece una aproximación potente para estudiar los mecanismos básicos de la adquisición y recaída de una adicción, así como para desarrollar tratamientos exitosos.

En general, se ha observado que la exposición al estrés conduce a disminuir el consumo de alimento y agua, inhibe la actividad exploratoria, suprime las respuestas motivadas apetitivas, incrementa las conductas ansiógenas y mejora o impide los aprendizajes aversivo y apetitivo (revisado en Steckler, 2005). En otras palabras, hay una priorización de las respuestas de estrés sobre otro tipo de conductas, y dichas respuestas reducen o eliminan los efectos negativos del estresor.

\section{El alcohol etílico (etanol)}

El compuesto químico etanol, conocido como alcohol etílico, es un agente psicofarmacológico complejo que ejerce un amplio rango de efectos en el organismo (calóricos, orosensoriales, etc.). Sin embargo, la vulnerabilidad al abuso del alcohol es principalmente influenciada por el balance entre sus efectos motivacionales. El etanol ejerce efectos reforzantes positivos y negativos, así como efectos aversivos agudos, derivados de sus propiedades reforzantes y aversivas, respectivamente. A continuación se exponen algunas de las cuestiones más importantes referentes a dichas propiedades, y su estudio experimental con animales.

\section{Propiedades reforzantes del etanol}

La mayor parte de las consecuencias reforzantes del etanol implica efectos postingestivos agradables mediados por la activación de centros de refuerzo del encéfalo medio. El refuerzo positivo mediado por etanol ha sido con frecuencia considerado el mecanismo clave que subyace al inicio y mantenimiento del consumo de alcohol (revisado en Pautassi et al., 2009). Esta droga facilita la liberación central de dopamina y de opioides endógenos, sistemas de neurotransmisores implicados en la evaluación hedónica de las drogas y de otros estímulos (Ericson, Molander, Lof, Engel y Soderpaim, 2003).

El etanol también reduce los estados centrales negativos como ansiedad y disforia (Wilson et al., 2004). Estos efectos reforzantes negativos determinarían las conductas de búsqueda y consumo de alcohol, y dichos efectos podrían estar asociados con un cambio en el control motivacional del reforzamiento inducido por etanol, de positivo a negativo. Es decir, el uso del alcohol podría estar predominantemente motivado, no por los efectos reforzantes del etanol, sino por su habilidad para contrarestar el estrés y disminuir la ansiedad y los estados emocionales depresivos (Koob, 2006; Koob y LeMoal, 2008).

A pesar de estar mediados por diferentes mecanismos cerebrales, los reforzamientos positivo y negativo inducidos por el etanol comparten consecuencias conductuales similares. Ambas fuentes de reforzamiento incrementan la probabilidad de aparición de conductas dirigidas a la consecución del etanol e inducen preferencias condicionadas a estímulos que predicen sus consecuencias placenteras (revisado en Cunningham, Fidler y Hill, 2000; Pautassi et al., 2009; Sanchis-Segura y Spanagel, 2006).

El condicionamiento de preferencia por un lugar (CPL) ha sido, quizá, el paradigma más empleado para evaluar el aprendizaje motivacional mediado por el etanol (revisado en Tzschentke, 1998). En este paradigma, los emparejamientos entre las consecuencias farmacológicas del etanol y los estímulos táctiles o visuales pueden resultar en un aprendizaje asociativo apetitivo medido como el incremento en la preferencia por dichos estímulos condicionados (Cunningham, Clemans et al., 2002; Escarabajal, De Witte y Quertemont, 2003; Quertemont, Escarabajal y De Witte, 2003).

Cabe mencionar, no obstante, que dada la naturaleza del etanol, y su capacidad para inducir efectos reforzantes y aversivos, es frecuente obtener aprendizaje de aversión, en lugar de preferencia, por un lugar en ratas (no tanto en ratones), sobre todo si la dosis de etanol es superior a 1 gr/kg (ver Green y Grahame, 2008). No obstante, las condiciones bajo las cuales los animales desarrollan preferencia o aversión por un lugar dependen de muchos factores, como la vía de administración (intaperitoneal, intragástrica, consumo forzado, etc.), cepa o línea de rata, experiencia con la droga previa al condicionamiento (Busse, Lawrence y Riley, 2005; Cunningham y Gremel, 2006), el tipo de aparato y procedimiento (Fidler, Bakner y Cunningham, 2004), y la temperatura a la cual están expuestos los animales (revisado en Kamenetzky, Cuenya, Pedrón y Mustaca, 2007).

El otro método comúnmente empleado para estudiar el reforzamiento consiste en usar procedimientos operantes. En estos, se requiere una acción instrumental aprendida para producir un determinado resultado, y la variable dependiente usada suele ser la cantidad consumida, o la frecuencia con la que se da la conducta encaminada a la consecución del resultado deseado. Los procedimientos operantes pueden ser usados para evaluar las propiedades reforzantes del etanol, e incluyen la autoadministración oral operante y la autoadministración intravenosa (revisado en Green y Grahame, 2008; y Sanchis-Segura y Spanagel, 2006).

El consumo libre de etanol también se puede considerar un índice de la eficacia reforzante de esta sustancia. La aceptación inicial del alcohol, en humanos y en ratas genéticamente heterogéneas, es modulada por el aparente disgusto que provoca el sabor del alcohol, por lo que se ha propuesto 
que las características sensoriales del alcohol constituyen una "barrera gustativa" (revisado en Pautassi et al., 2009), de manera que, las ratas sólo comienzan a consumir etanol si es presentado a bajas concentraciones o en situación de privación de fluidos. Sin embargo, una concentración demasiado baja no tendría efectos significativos. En general, se considera que, las concentraciones entre 8 y $12 \%$ son adecuadas, y las concentraciones de etanol por debajo de $4 \%$ ( $\mathrm{v} / \mathrm{v})$ no son farmacológicamente pertinentes. Por lo tanto, la concentración de alcohol es un tema crítico en estos procedimientos. Este problema ha sido parcialmente solucionado gracias a la selección genética (ver Green y Grahame, 2008; Pautassi et al., 2010), la exposición a series de concentraciones incrementadas de etanol (Aragón, Sternklar y Amit, 1985; Shoaib y Almeida, 1996), y a procedimientos como el "enmascaramiento por sucrosa", que permiten superar la "barrera gustativa" que representa el disgusto innato que provocan las propiedades orosensoriales del alcohol (Samson, 1986; ver también Boyle, Smith, Spivak y Amit, 1994; Cunningham et al., 2000).

Los efectos reforzantes negativos, anti-ansiedad, del etanol pueden ser evaluados a través del uso de paradigmas de condicionamiento conductual o paradigmas basados en conductas no condicionadas. Los modelos basados en conductas incondicionadas evalúan los efectos del etanol cuando los animales son expuestos a estímulos que elicitan por sí mismos ansiedad. Este es el caso de las tareas de aproximación-evitación como el laberinto arena iluminado, el test luz/oscuridad y el laberinto elevado (Wilson et al., 2004). Los animales normalmente tienden a evitar espacios abiertos iluminados, pero aquellos a los que se les administra etanol, normalmente muestran un aumento en la exploración de dichos espacios (revisado en Camarini et al., 2010). Otras tareas que se han empleado para evaluar los efectos antiansiedad o de tensión-reducción del alcohol son el test de interacción social, el contraste negativo, el test de Vogel, o el test de Geiller-Seifer, en las que el alcohol tiene efectos anticonflicto (revisado en Koob y LeMoal, 2006).

\section{Propiedades aversivas del etanol}

Los efectos aversivos del etanol incluyen alteraciones fisiológicas periféricas como, molestias gastrointestinales (Adinoff, Iranmanesh, Veldhius y Fisher, 1998) y reacciones autonómicas (Duranceaux et al., 2006), así como efectos sobre el sistema nervioso central (SNC) (revisado en Pautassi et al., 2009).

Los efectos postabsortivos negativos del etanol pueden servir como estímulos incondicionados (EI) aversivos, disminuyendo la preferencia por estímulos asociados con la administración de la droga (Cunningham, Clemans et al., 2002). Se ha demostrado que el condicionamiento aversivo gustativo (CAG) es uno de los paradigmas más fiables y sensibles para evaluar los efectos aversivos del etanol. Los roedores que acceden a un estímulo gustativo (EC) seguido de etanol exhiben subsecuentemente una evitación del sabor asociado con la droga. Concretamente, se ha observado que sujetos maduros expresan CAG inducido por etanol después de dos o tres ensayos de condicionamiento y cuando se usan como EI dosis de etanol moderadas y altas (1.0-5.0 gr $/ \mathrm{kg})$. Las dosis bajas de etanol $(0.5-1.0 \mathrm{gr} / \mathrm{kg})$, por el contrario, no parecen inducir CAG (revisado en Liu, Showalter, y Grigson, 2009).

Entre los mecanismos que probablemente subyacen a la habilidad del etanol para inducir CAG, dos han recibido sustancial apoyo empírico: la hipotermina inducida por la droga y la inducción de efectos eméticos a través de la activación de los quimioreceptores del troncoencéfalo (revisado en Pautassi et al., 2009). En contraste, otros autores han sugerido la posibilidad de que el CAG inducido por etanol sea en realidad una forma de contraste anticipatorio (el rechazo a ingerir un estímulo gustativo que predice la ocurrencia de un EI reforzante, como el etanol) (Sorge, Fudge y Parker, 2002; revisado en Grigson, 1997). No obstante, existen otros resultados que no apoyan esta hipótesis (revisado en Green y Grahame, 2008). Cunningham, Tull, Rindal y Meyer (2002), por ejemplo, han comprobado la existencia de un efecto diferencial de la preexposición al ethanol sobre los niveles de condicionamiento de preferencia (CPL) y aversión (CAL) por un lugar inducido por etanol, por lo que los autores consideran que ambos tipos de condicionamiento no pueden estar causados por un efecto reforzante monovalente del etanol, sino por efectos motivacionales opuestos independientes de la droga. Estos datos podrían ser extrapolados, en cierta medida, al paradigma de CAG, puesto que existen datos que reflejan una correlación positiva entre el CAG y el CAL causados por una administración intraperitoneal de etanol tras la exposición al EC (Cunningham y Ignatoff, 2000).

En un estudio reciente, Liu et al. (2009) han contribuido a esclarecer si la supresión de la ingesta de un EC inducida por etanol se debe a los efectos reforzantes de la droga (efecto de contraste anticipatorio) o a sus efectos aversivos (CAG propiamente dicho). Los autores indican que el etanol es capaz de inducir CAG por las propiedades reforzantes o aversivas de la droga, lo que estaría determinado principalmente por la dosis de etanol empleada. Según Liu y sus colaboradores, con dosis elevadas $(1.5 \mathrm{gr} / \mathrm{kg})$ el CAG se sostendría por los efectos aversivos del etanol, ya que, en este caso, las lesiones del tálamo gustativo (que no interrumpen el CAG inducido por drogas eméticas, como cloruro de litio, pero sí eliminan el efecto de contraste anticipatorio) no interrumpieron el aprendizaje; en cambio, dichas lesiones sí lo hicieron con dosis inferiores de etanol $(0.75 \mathrm{gr} / \mathrm{kg})$.

Por tanto, a pesar de la controversia existente acerca de la naturaleza de la supresión de la ingesta de un EC inducida por etanol en el paradigma de CAG, los datos presentados nos llevan a interpretar tal supresión en términos de CAG y no en términos de contraste anticipatorio.

Por último, cabe mencionar la existencia de diferencias, determinadas genética y/o ambientalmente, en la sensibi- 
lidad a los efectos aversivos del etanol, y éstas podrían, por tanto, influir sobre la probabilidad de búsqueda y consumo de etanol, como se extrae de diversas investigaciones realizadas con roedores (revisado en Cunningham et al., 2000; Broadbent et al., 2002; Escarabajal, 2003; y, Green y Grahame, 2008) y también con humanos (véase Duranceaux et al., 2006). En general, se ha observado que aquellos individuos que muestran mayores índices de CAG tienen una menor preferencia por el alcohol. En definitiva, la mayor sensibilidad a los efectos aversivos del etanol podría considerarse un efecto protector frente al alcoholismo (Broadbent et al., 2002).

Estudio de los procesos de tolerancia y/o sensibilización a los efectos motivacionales del etanol

La tolerancia y la sensibilización son las dos formas más estudiadas de neuroadaptación producida por la exposición repetida a una droga. Estos procesos reflejan un intento del organismo de adaptarse a los efectos agudos y/o crónicos de las drogas de abuso, que son contrarrestados por los cambios homeostáticos de los fenómenos de tolerancia y/o sensibilización. Los dos procesos han sido implicados en las discusiones teóricas sobre el incremento progresivo de la ingesta de etanol exhibida por personas que abusan del alcohol. Por ejemplo, dicho incremento se ha atribuido algunas veces a un efecto de tolerancia a los efectos reforzantes del etanol, lo que supone que los individuos han de incrementar las dosis consumidas para mantener el efecto deseado de la droga. Por otra parte, el incremento de consumo de etanol también podría ser explicado en términos de tolerancia a los efectos inhibitorios y aversivos de la droga, que inicialmente limitan el consumo de la misma, pero que van desapareciendo con la exposición repetida, permitiendo por tanto mayores niveles de consumo. Finalmente, el incremento progresivo en el consumo de etanol ha sido explicado como un efecto de sensibilización a los efectos reforzantes de la droga (revisado en Cunningham, Tull et al., 2002).

Se han desarrollado una serie de pruebas conductuales con la finalidad de estudiar estos procesos de tolerancia y sensibilización a los efectos motivacionales de las drogas de abuso. Respecto a las pruebas diseñadas para evaluar el desarrollo de sensibilización cabe destacar el modelo de sensibilización conductual. El término "sensibilización" se refiere al incremento en una respuesta tras la ocurrencia repetida del estímulo que promueve la mencionada respuesta. La sensibilización en la investigación sobre las drogas de abuso ha sido principalmente estudiada con respecto a la actividad locomotora. Así, se ha observado que la habilidad de las drogas adictivas de incrementar la actividad locomotora después de la administración aguda, es progresivamente facilitada por la exposición repetida a la droga. Este robusto fenómeno, observado en una gran variedad de especies, ha sido reconocido como un buen modelo de los cambios neuroadaptativos que subyacen a la adicción a las drogas, y se considera generalmente como un índice de las propiedades motivacionales de incentivo del etanol (revisado en Camarini et al., 2010). De hecho, este fenómeno está implicado en el cambio de la saliencia del incentivo, desde un "deseo" hacia la droga, al deseo patológico o craving, que definiría su uso compulsivo (ver Kreek y Koob, 1998).

Por otra parte, procedimientos como el condicionamiento aversivo gustativo, o a un lugar, han sido empleados no sólo para evaluar la tolerancia a los efectos aversivos de una droga tras la exposición repetida a la misma, sino también los procesos de tolerancia o sensibilización a los efectos reforzantes de la sustancia. Por ejemplo, Cunningham, Tull et al. (2002), realizaron un estudio con objeto de examinar los efectos de dos formas de pre-exposición al alcohol (distal y proximal, inyectando etanol de forma intraperitoneal en ambos casos) sobre los efectos reforzantes y aversivos del etanol, mediante condicionamiento a un lugar en ratones. Los resultados obtenidos mostraron que tanto la pre-exposición distal (48 h. antes) como la proximal (65 minutos antes) interfirieron con la adquisición del CAL inducido por etanol, mientras que ninguna de las formas de pre-exposición al etanol influyó sobre la adquisición del CPL. Estos resultados podrían sugerir que incrementos en el consumo de etanol observados tras exposición crónica a etanol son causados probablemente por tolerancia a los efectos aversivos del etanol, y no por sensibilización a sus efectos reforzantes. Cabe mencionar, no obstante, que los resultados obtenidos por Shoaib y Almeida (1995), empleando ratas, contrastan con el patrón de resultados que acabamos de exponer, ya que no hallaron un efecto de tolerancia a los efectos aversivos del etanol, evaluados mediante CAG, tras el consumo voluntario de la sustancia.

Parece evidente, por tanto, que factores como el método de pre-exposición a etanol elegido, o las diferencias entre especies, podrían determinar la aparición de los efectos de tolerancia o sensibilización, por lo que este tema debería ser investigado con mayor profundidad.

\section{Diferencias sexuales y evolutivas en los efectos mo- tivacionales del etanol}

Generalmente, en los estudios que examinan los niveles de consumo de etanol en roedores, se ha comprobado que las hembras maduras exhiben un mayor consumo de etanol que los machos de su misma edad (Cailhol y Mormede, 2001; Le, Israel, Juzytsch, Quan y Harding, 2001; Chester, de Paula Barrenha y DeMaria, 2006). Estas diferencias sexuales se han observado usando los modelos de $24 \mathrm{~h}$ de acceso a etanol (Doremus, Brunell, Rajendran y Spear, 2005), de acceso limitado a etanol en situaciones de elección de dos botellas (Le et al., 2001; Vetter-O’Hagen, Varlinskaya y Spear, 2009) y los de auto-administración operante (Blanchard y Glick, 1995; Blanchard, Steindorf, Wang y Glick, 1993), tanto en ratas no consanguíneas (p.ej., Lancaster, Brown, Coker, Elliott, y Wren, 1996), como en ratas y ratones seleccionados por su alto o bajo nivel de consumo de etanol (p.ej., Le et al., 2001; Chester et al., 2006). 
Vetter-O’Hagen et al. (2009), examinaron y ampliaron datos en relación con esta cuestión, utilizando un modelo de acceso limitado $(2 \mathrm{~h})$ a etanol. Sus resultados indicaron que el consumo de etanol varía, tanto en función del sexo como de la edad, aunque las diferencias sexuales que emergen en cada edad son opuestas. Concretamente, los datos indicaron que los machos adolescentes consumen más etanol que las hembras adolescentes y que las adultas de ambos sexos, mientras que, en consonancia con las observaciones de otros grupos de investigación, se observó que las hembras adultas generalmente consumen más etanol que los machos adultos.

Cabe mencionar, no obstante, que los hallazgos obtenidos por García-Burgos, González, Manrique y Gallo (2009), usando un modelo de acceso ilimitado a etanol $24 \mathrm{~h}$., si bien parecen indicar un mayor consumo de etanol por parte de ratas adolescentes frente a ratas adultas, no reflejan diferencias determinadas por el sexo de los sujetos, en ninguna de las dos etapas evolutivas estudiadas.

Estos datos reflejan un cierto grado de divergencia respecto a los niveles de consumo de etanol dependiendo del sexo de los sujetos, por lo que consideramos que debería ampliarse la investigación sobre esta cuestión, quizá cuidando especialmente aspectos como la edad exacta de los sujetos o el tipo de modelo de acceso a etanol empleado.

En cambio, los datos referentes a los niveles de consumo de sujetos adolescentes frente a los de sujetos adultos, parecen caracterizarse por contar por una alta consistencia. De hecho, la mayor parte de los estudios, realizados tanto con humanos (revisado en Song et al., 2007), como con animales (ver Anderson, Varinskaya y Spear, 2010; Doremus et al., 2005; Garcia-Burgos et al., 2009; Song et al., 2007; Vetter-O’Hagen et al., 2009), indican que los individuos adolescentes consumen mayores cantidades de alcohol que los adultos, probablemente debido a diferencias en los niveles hormonales y de neuroadaptación.

A nivel conductual, la adolescencia se caracteriza por una mayor búsqueda de sensaciones, de conductas de riesgo, impulsividad, ansiedad, etc. Estas características se asocian con cambios en la secreción de esteroides gonadales y de hormonas relacionadas con el estrés, que podrían explicar el patrón de inciación de consumo de etanol y de otras drogas. Igualmente, el desarrollo relativamente tardío de los circuitos de la corteza prefrontal implicados en la realización de juicios y en el control inhibitorio, puede subyacer a la propensión de los adolescentes a la impulsividad y a ignorar las consecuencias negativas de su conducta, lo que puede incrementar el riesgo de abuso de alcohol (ver Guerri y Pascual, 2010).

Se han recopilado toda una serie de evidencias que señalan la existencia de un patrón general de reactividad al alcohol distintivo de los sujetos adolescentes frente a los adultos (ver, por ejemplo, Guerri y Pascual, 2010). La investigación en este campo con humanos es problemática, y está altamente limitada por constricciones éticas y legales. Sin embargo, se ha conseguido un gran avance en las últimas décadas gracias al empleo de modelos animales. Los datos recopilados a partir de estas investigaciones parecen indicar que los adolescentes, en general, son menos sensibles a una amplia gama de efectos sedativos (narcosis, coordinación motora, etc.), aversivos y estimulantes del etanol (por ejemplo, sensibilización conductual) (Vetter-O’Hagen et al., 2009; Anderson et al., 2010), pero sí muestran mayor afectación cognitiva después del tratamiento con la droga, y mayor sensibilidad a los efectos positivos reforzantes del etanol, evaluados mediante CPL (revisado en Pautassi et al., 2010).

Por ejemplo, en un estudio con ratas cuyas edades variaron entre $25,35,45$ y 60 días, y fueron inyectadas con $0.0,0.2,0.5,1.0$ y $2.0 \mathrm{~g} / \mathrm{kg}$ de etanol, se halló que los animales más jóvenes mostraron CPL en la dosis más baja y CAL en dosis altas. Las ratas adolescentes (45 días) mostraron CPL con dos dosis moderadas y CAL con la dosis alta, los animales de 35 y 60 días no mostraron CPL bajo ninguna dosis, y los animales de 60 días mostraron una aversión creciente a medida que se aumentaba la dosis (Philpot, Badanich y Kirstein, 2003). Estos resultados sugieren que los animales jóvenes son más propensos a desarrollar CPL y, por tanto, parecen ser más sensibles a los efectos reforzantes del etanol que los adultos.

Por otra parte, Vetter-O'Hagen et al. (2009) han aportado datos que parecen poner de manifiesto que ratas adolescentes, tanto machos como hembras, requieren mayores dosis de etanol para inducir CAG que las ratas adultas. En la misma línea, Anderson et al. (2010) han informado que las ratas adolescentes empleadas en su estudio, no sólo requirieron una mayor dosis de etanol para inducir $C A G$, sino también un mayor número de emparejamientos entre el estímulo condicionado y el etanol. Estos datos parecen poner de manifiesto la existencia de una insensibilidad específica de los individuos adolescentes a las propiedades aversivas del etanol capaces de inducir CAG.

Teniendo en cuenta estos datos, se podría afirmar que los sujetos adolescentes son más sensibles a los efectos positivos del etanol y menos sensibles a las consecuencias aversivas de la droga. Este distintivo patrón de respuesta a los efectos motivacionales del alcohol podría situar a los adolescentes en una situación de riesgo para el desarrollo de trastornos relacionados con el alcohol (ver Spear y Varlinskaya, 2010). No obstante, la cantidad de investigación recopilada en apoyo de esta afirmación es aún limitada, por lo que es necesario investigar más profundamente esta cuestión para alcanzar una conclusion definitiva.

\section{Relación entre estrés y efectos motivacionales del alcohol}

Como se comentó en apartados previos, han sido numerosos los modelos animales desarrollados para intentar determinar si el estrés es un factor causal en el desarrollo de una adicción. Gracias a este tipo de estudios existe un amplio conjunto de datos, neuroanatómicos, neuroquímicos 
y neuroconductuales, acerca de las interacciones recíprocas entre los efectos del estrés y los de las drogas de abuso.

Respecto a las interrelaciones entre los efectos del estrés y los del etanol, se ha demostrado, por una parte, que esta sustancia es capaz de eliminar las reacciones inducidas por sonido, normalizar la conducta en situaciones de conflicto, revertir los efectos neuroquímicos del estrés, y restablecer la actividad locomotora normal después del estrés (revisado en Hegarty y Vogel, 1993; Koob y LeMoal, 2006). Así mismo, se ha demostrado que el estrés incrementa la ingesta voluntaria de etanol (i.e., Vengeliene et al., 2003), aunque existen resultados contradictorios dependiendo del modelo de estrés empleado, o de determinados parámetros experimentales dentro de un modelo concreto, así como del procedimiento de consumo de etanol utilizado (ver Shina, 2005; Zimmermann, Blomeyer, Laucht, y Mann, 2007; y, Pautassi et al., 2010 para revisión sobre el tema). Por otra parte, se ha observado que el estrés facilita el CPL (revisado en Lu et al., 2003; Sperling, Gomes, Sypek, Carey y McLaughlin, 2010) y minimiza el CAL inducido por $1.0 \mathrm{~g} / \mathrm{kg}$ de etanol (Funk, Vohra y Le, 2004).

Son varias las hipótesis que se pueden elaborar acerca de cómo los efectos del etanol pueden interactuar con los efectos del estrés, determinando así la respuesta conductual de los individuos ante la droga en situaciones de estrés. Así, se puede hipotetizar que el etanol actúa activando el sistema de refuerzo cerebral (principalmente dopaminérgico), directa e indirectamente (a través de la liberación de opioides endógenos), minimizando así los efectos aversivos del estrés; o bien, se podría pensar que el etanol es capaz de actuar como reforzador negativo, reduciendo la tensión provocada por el estrés, probablemente a través de la amortiguación de la activación dopaminérgica inducida por el estrés, de forma directa o indirecta (a través de la activación del sistema inhibitorio GABAérgico). De hecho, se ha informado de la existencia de diferentes efectos dosis-dependientes del etanol sobre ratas sometidas a estrés. Concretamente, se ha comprobado que el etanol es capaz de antagonizar los incrementos de DA inducidos por estrés cuando se administra en dosis altas ( $2 \mathrm{~g} / \mathrm{kg}$ i.p.), mientras que dosis bajas $(0,5 \mathrm{~g} / \mathrm{kg}$. i.p.) incrementan de forma significativa los niveles de DA y de ácido di-hidroxi-fenil-acético (DOPAC), en la corteza prefrontal medial (CPM) (Hegarty y Vogel, 1993). Se podría afirmar, por tanto, que el etanol, a dosis altas, puede antagonizar los efectos del estrés en el SNC, haciendo de la conducta de ingesta de alcohol una respuesta de afrontamiento alternativa al estrés que puede conducir al abuso de esta sustancia y, por tanto, al alcoholismo (revisado en Shina, 2005). El hallazgo de que el etanol administrado en bajas dosis potencia la liberación de DA apoyaría, por otra parte, la idea de que los incrementos en DA inducidos por etanol contrarrestarían los efectos aversivos del estrés, dado que la activación del sistema dopaminérgico está relacionada con las propiedades reforzantes de las drogas de abuso, incluyendo al etanol (revisado en Hegarty y Vogel, 1993).
En definitiva, en situaciones de exposición a estrés, las propiedades reforzantes del etanol (positivas o negativas) podrían resultar incrementadas. En este sentido, Koob y LeMoal (2008) han sugerido que el estrés conduce a neuroadaptaciones en los circuitos cerebrales de refuerzo generando una mayor sensibilidad a las propiedades reforzantes de las drogas e incrementando, por tanto, su uso compulsivo. De hecho, existe evidencia, procedente de estudios realizados con humanos, de la existencia de diferencias en la respuesta subjetiva a drogas como el alcohol, los psicoestimulantes y la nicotina. Datos recientes atribuyen tales diferencias a la variabilidad en la respuesta del sistema dopaminérgico mesolímbico a la administración aguda de una droga y, tal variabilidad, podría estar determinada no sólo por factores genéticos, sino también por factores ambientales, como el estrés. En esta cuestión podría estar la clave de la transición de un uso circunstancial de una droga a un uso crónico y regular de la misma (revisado en Shina, 2005).

Por otra parte, cabe preguntarse si el estrés tiene también la capacidad de minimizar los efectos aversivos del etanol, como parece indicar el hecho de que el estrés sea capaz de reducir el aprendizaje de aversión a un lugar inducido por etanol (Funk et al., 2004; Funk, Hurding, Juzytsch y Le, 2005), contribuyendo también con ello al incremento de la sensibilidad a las propiedades reforzantes de las drogas, a través de un mecanismo neurobiológico similar o distinto.

Desafortunadamente, aunque existe un abundante conjunto de datos que indican que el estrés modula la adquisición de un problema de adicción, aún no se puede determinar con certeza cuáles son los mecanismos neurobiológicos específicos que describen mejor la interacción entre el etanol y el estrés en el SNC, y en qué medida contribuyen tales mecanismos al inicio, mantenimiento y la recaída del alcoholismo. Sin duda, la investigación en esta línea se hace necesaria de cara a desarrollar terapias efectivas para la prevención y el tratamiento del alcoholismo.

\section{Conclusiones}

La investigación clínica indica que el estrés es un factor de riesgo en el desarrollo de una adicción. Aún se desconoce cómo puede el estrés influir en el inicio, mantenimiento y recaída de la conducta alcohólica. Una de las hipótesis que se baraja es la posibilidad de que el estrés pueda influir sobre la preferencia de consumo de alcohol a través de su efecto sobre la sensibilidad diferencial a las propiedades reforzantes y aversivas del alcohol y que, por supuesto, tal modulación pueda depender de la predisposición genética particular, de la historia previa de consumo de la droga, del sexo, de la etapa evolutiva, etc., de cada individuo (ver Pautassi et al., 2010).

Combinar todos estos factores a nivel experimental, así como investigar las contribuciones específicas de cada uno de ellos a la adquisición de un problema de alcoholismo resulta una difícil tarea. Algunos estudios han intentado, no 
obstante, acercarse al problema estudiando las interacciones entre algunos de estos factores.

Por ejemplo, los estudios desarrollados con ratas seleccionadas por sus niveles de preferencia y consumo de etanol (ver, por ejemplo, Roma, Rinker et al., 2008, y Vengeliene et al, 2003), o por sus patrones de reactividad emocional (ver Guitart-Masip et al., 2006), que son sometidas a distintas situaciones de estrés, son una manera de acercarse al estudio de las interacciones entre la predisposición genética y las condiciones ambientales.

Roma, Rinker et al. (2008), realizaron un estudio con el objetivo de determinar las contribuciones relativas de los factores genéticos y ambientales a los efectos aversivos condicionados del etanol, empleando para ello ratas hembra de las cepas Fischer 334 y Lewis, genéticamente seleccionadas por su conducta diferencial relacionada con el alcohol. Los autores observaron que individuos aparentemente idénticos para determinadas conductas pueden diferir en su susceptibilidad a la influencia y modulación de factores ambientales sobre los efectos motivacionales de las drogas de abuso.

En este sentido, se ha sugerido que las sublíneas de ratas RHA y RLA (ratas romanas consanguíneas de alta y baja evitación; en inglés, Roman High Avoidance -RHA- y Roman Low Avoidance -RLA-, respectivamente), genéticamente seleccionadas por sus diferencias en reactividad emocional, podrían ser un modelo de laboratorio válido para estudiar los perfiles divergentes de búsqueda de novedad y de sustancias reforzantes, así como de la actividad diferencial central del sistema dopaminérgico en un amplio rango de situaciones experimentales. En efecto, comparadas con las ratas RLA, las RHA muestran: (1) mayores niveles de conducta exploratoria en test de búsqueda de novedad; (2) mayor preferencia por alcohol, así como por soluciones de sacarina y de quinina; (3) mayores respuestas dopaminérgicas

\section{Referencias}

Adinoff, B., Iranmanesh, A., Veldhius, J., y Fisher, L. (1998). Disturbances of the stress response: the role of the HPA axis during alcohol withdrawal and abstinence. Alcohol Health Research, 22, 67-72.

Alonso, J., y Navarro, E. (1993). El estrés. Medicina clínica, 100, 26-28.

Anderson, R. I., Varinskaya, E. I. y Spear, L. P. (2010). Ethanol-induced conditioned taste aversion in male sprague-dawley rats: impact of age and stress. Alcohol: Clinical and Experimental Research, 34(12), 2106-2115. doi: 10.1111/j.1530-0277.2010.01307.x.

Aragón, C., Sternklar, G., y Amit, Z. (1985). A correlation between voluntary ethanol consumption and brain catalase activity in the rat. Alcohol, 2, 353-356. doi: 10.1016/0741-8329(85)90074-6.

Blanchard, B. A. y Glick, S. D. (1995). Sex differences in mesolímbico dopamine responses to ethanol and relationship to ethanol intake in rats. Recent Developments in Alcoholism, 12, 231-241.

Blanchard, B. A., Steindorf, S., Wang, S. y Glick, S. D. (1993). Sex differences in ethanol-induced dopamine release in nucleus accumbens and in ethanol consumption in rats. Alcoholism: Clinical and Experimental Research, 17, 968-973. doi: 10.1111/j.1530-0277.1993.tb05650.x.

Boyle, A. E., Smith, B. R., Spivak, K. y Amit, Z. (1994). Voluntary ethanol consumption in rats: the importance of the exposure paradigm in determining the final intake outcome. Behavioral Pharmacology, 5, 502512. doi: 10.1097/00008877-199408000-00011. mesolímbicas y mesocorticales al estrés y a las sustancias adictivas, incluyendo el etanol. Además, las ratas RHA son menos sensibles a los efectos hipnóticos del alcohol (revisado en Guitart-Masip et al., 2006). Guitart-Masip et al. (2006), investigaron, además, si la fuerte preferencia por el etanol exhibida por las RHA en comparación con las RLA (Fernández-Teruel et al., 2002), podía estar relacionada con la menor sensibilidad de la cepa RHA a los efectos del alcohol. Los resultados obtenidos en este estudio revelaron que las ratas RHA pueden representar un modelo animal único para estudiar la predisposición al alcoholismo en humanos, ya que estas ratas muestran una menor sensibilidad a los efectos estimulantes e hipnóticos de una baja dosis de etanol que las ratas RLA.

En general, estos hallazgos parecen indicar que factores genéticos y ambientales interactúan para modelar las respuestas al alcohol, y que los individuos pueden diferir en su receptividad a la modulación epigenética de los sistemas subyacentes a las respuestas motivacionales ante dicha sustancia. Determinar las bases neurobiológicas de esta susceptibilidad y resistencia en modelos animales, y en humanos, permanece como un objetivo prioritario de investigación.

En definitiva, aunque existen datos acerca de las relaciones entre el estrés y los efectos motivacionales del etanol, atendiendo a diferentes cuestiones como la predisposición genética de los sujetos, consideramos que es necesario ampliar la investigacion en este campo, empleando una gran variedad de modelos animales de estrés, de especies y cepas de animales, y de pruebas conductuales para evaluar los efectos reforzantes y/o aversivos del etanol, promoviendo la interacción entre las diferentes aproximaciones y procurando trasladar los hallazgos obtenidos a partir de la investigacion con animales a la investigación epidemiológica, con objeto de poder comprender mejor los procesos que subyacen al inicio, mantenimiento y la recaida de una adicción al alcohol.

Briand, L. y Blendy, J. A. (2010). Molecular and genetic substrates linking stress and addiction. Brain Research, 1314, 219-234. doi: 10.1016/j.brainres.2009.11.002.

Broadbent, J., Muccino, K. J., y Cunningham, C. L. (2002). Ethanol-induced conditioned taste aversion in 15 inbred mouse strain. Behavioral Neuroscience, 116, 138-148. doi: 10.1037//0735-7044.116.1.138.

Busse, G. D., Lawrence, E. T. y Riley, A. L. (2005). The effects of alcohol preexposure on cocaine, alcohol and cocaine/alcohol place conditioning. Pharmacology, Biochemistry \& Behavior, 81, 459-465. doi: 10.1016/j.pbb.2005.04.003.

Caihol, S. y Mormede, P. (2001). Sex and strain differences in ethanol drinking: effects of gonadectomy. Alcohol: Clinical and Experimental Research, 25, 594-599. doi: 10.1111/j.1530-0277.2001.tb02255.x.

Camarini, R., Pautassi, R., Méndez, M., Quadros, I. M., Souza-Formigoni, M. L. y Boerngen-Lacerda R. (2010). Behavioral and neurochemical studies in distinct animal models of ethanol's motivational effects. Current Drug Abuse Reviews, 3(4), 1-17. doi: 10.2174/1874473711003040205.

Chester, J. A., de Paula Barrenha, G., y DeMaria, A. (2006). Different effects of stress on alcohol drinking behavior in male and female mice selectively bred for high alcohol preference. Alcohol \& Alcoholism, 41, 44-53. doi: $10.1093 /$ alcalc/agh242. 
Cunningham, C. L., Clemans, J., y Fidler, T. (2002). Injection timing determines wthether intragastric ethanol produces conditioned place preference or aversion in mice. Pharmacology, Biochemestry \& Behavior,72(3), 659-68. doi: 10.1016/S0091-3057(02)00734-7.

Cunningham, C. L., Fidler, T. y Hill, K. G. (2000). Animal models of alcohol's motivational effects. Alcohol Research \& Health, 24(2), 85-92.

Cunningham, C. L. y Gremel, C. M. (2006). Proximal ethanol pretreatment interferes with acquisition of ethanol-induced conditioned place preference. Pharmacology, Biochemistry \& Behavior, 85, 612- 619. doi: 10.1016/j.pbb.2006.10.016.

Cunningham, C. L. y Ignatoff, D. L. (2000). Genetic differences in ethanolinduced conditioned place aversion. Alcoholism, Clinical and Experimental Research, 24: 57A.

Cunningham, C. L., Tull, L. E., Rindal, K. E., y Meyer, P. J. (2002). Distal and proximal pre-exposure to ethanol in the place conditioning task: tolerance to aversive effect, sensitization to activating effect, but no change in rewarding effect. Psychopharmacology, 160, 414-424. doi: 10.1007/s00213-001-0990-1.

Doremus, T. L., Brunell, S. C., Rajendran, P. y Spear, L. P. (2005). Factors influencing elevated ethanol consumption in adolescent relative to adult rats. Alcohol: Clinical and Experimental Research, 29, 1796-808. doi: 10.1097/01.alc.0000183007.65998.aa

Duranceaux, N., Schuckit, M. A., Eng, M. Y., Robinson, S. K., Carr, L. G. y Wall, T. L. (2006). Associations of variations in alcohol dehydrogenase genes with the level of response to alcohol in non-Asians. Alcoholism, Clinical and Experimental Research, 30, 1470-1478. doi: 10.1111/j.15300277.2006.00178.x.

Ericson, M., Molander, A., Lof, E., Engel, J. y Soderpaim, B. (2003). Ethanol elevates accumbal dopamine levels via indirect activation of ventral tegmental nicotinic acetylcholine receptors. European Journal of Pharmachology, 467, 85-93. doi: 10.1016/S0014-2999(03)01564-4.

Escarabajal, M. D. (2003). Alteraciones genéticas relacionadas con el alcoholismo. Revista de Neurología, 37 (5), 471-480.

Escarabajal, M. D., De Witte, P. y Quertemont, E. (2003). Role of acetaldehyde in ethanol-induced conditioned taste aversión in rats. Psychopharmacology, 167, 130-136. doi: 10.1007/s00213-003-1427-9.

Fernández-Teruel, A., Driscoll, P., Gil, L., Aguilar, R., Tobeña, A. y Escorihuela, R. (2002). Enduring effects of environmental enrichment on novelty seeking,saccharin and ethanol intake in two rat lines (RHA/Verh and RLA/Verh) differing in incentive-seeking behavior. Pharmacology, Biochemistry and Behavior, 73, 225-231. doi: 10.1016/S00913057(02)00784-0.

Fidler, T. L., Bakner, L. y Cunningham, C. L. (2004). Conditioned place aversion induced by intragastric administration of ethanol in rats. Pharmacology, Biochemistry \& Behavior, 77, 731-743. doi: 10.1016/j.pbb.2004.01.010.

Fuchs, E. y Flügge, G. (2003). Chronic social stress: effects on limbic brain structures. Physiology \& Behavior, 79, 417-427. doi: 10.1016/S00319384(03)00161-6

Funk, D., Hurding, S., Juzytsch, W. y Le, A. D. (2005). Effects of unconditioned and conditioned social defeat on alcohol selfadministration and reinstatement of alcohol seeking in rats. Psychopharmacology, 183, 341-349. doi: 10.1007/s00213-005-0194-1.

Funk, D., Vohra, S., y Le, A. (2004). Influence of stressors on the rewarding effects of alcohol in Wistar rats: studies with alcohol deprivation and place conditioning. Psychopharmacology, 176, 82-87. doi: 10.1007/s00213004-1859-x.

Gambrana, C. (2007). Experimental protocols for the study of stress in animals and humans. En S. Yehuda, y D. I. Mostofsky (Eds.), Nutrient, Stress and Medical Disorders (pp. 21-35). New Jersey: Humana Press Inc.

García-Burgos, D., González, F., Manrique, T. y Gallo, M. (2009). Patterns of Ethanol Intake in Preadolescent, Adolescent, and Adult Wistar Rats Under Acquisition, Maintenance, and Relapse-Like Conditions. Alcoholism: Clinical and Experimental Research, 33(4), 722-728. doi: 10.1111/j.1530-0277.2008.00889.x.

Green, A. S. y Grahame, N. J. (2008). Ethanol drinking in rodents: is freechoice drinking related to the reinforcing effects of ethanol?. Alcohol, 42(1), 1-11. doi: 10.1016/j.alcohol.2007.10.005.

Grigson, P. (1997). Conditioned taste aversions and drugs of abuse: a reinterpretation. Behavioral Neuroscience, 111(1), 129-136.
Guerri, C. y Pascual, M. (2010). Mechanisms involved in the neurotoxic, cognitive, and neurobehavioral effects of alcohol consumption during adolescence. Alcohol, 44, 15-26. doi: 10.1016/j.alcohol.2009.10.003.

Guitart-Masip, M., Gimenez-Llort, L., Fernández-Teruel, A., Cañete, T., Tobeña, A., Ove Ögren, S. O., Terenius, L. y Johansson, B. (2006). Reduced ethanol response in the alcohol-preferring RHA rats and neuropeptide mRNAs in relevant structures. European Journal of Neuroscience, 23, 531-540. doi: 10.1111/j.1460-9568.2005.04556.x.

Hegarty, A. A. y Vogel, W. H. (1993). Modulation of the stress response by ethanol in the rat frontal cortex. Pharmacology, Biochemistry and Behavior 45, 297-299. doi: 10.1016/0091-3057(93)90247-Q.

Herman, J. P. y Cullinan, W. E. (1997). Neurocircuitry of stress: central control of the hypothalamo-pituitary-adreno-cortical axis. Trends in Neuroscience, 20, 78-84. doi: 10.1016/S0166-2236(96)10069-2.

Kamenetzky, G. V., Cuenya, L., Pedrón, V. y Mustaca, A. E. (2007) Condicionamiento de lugar en ratas y etanol. International Journal of Psychology and Psychological therapy, 7 (3), 321-333.

Koob, G. (2006). The neurobiology of addiction: a neuroadaptational view relevant for diagnosis. Addiction, 101, 23-30. doi: 10.1111/j.13600443.2006.01586.x.

Koob, G. y LeMoal, M. (2008). Addiction and the brain antireward system. Annual Reviews of Psychology, 59, 29-53. doi: 10.1146/annurev.psych.59.103006.093548.

Kreek, M. J. y Koob G. F. (1998). Drug dependence: stress and dysregulation of brain reward pathways. Drug and Alcohol Dependence, 51, 23-47. doi: 10.1176/appi.ajp.2007.05030503.

Lancaster, F. E., Brown, T. D., Coker, K. L., Elliott, J. A. y Wren, S. B. (1996). Sex differences in alcohol preference and drinking patterns emerge during the early postpubertal period. Alcoholism: Clinical and Experimental Research, 20, 1043-1049. doi: 10.1111/j.15300277.1996.tb01945.x.

Le, A. D., Israel, Y., Juzytsch, W., Quan B y Harding S. (2001). Genetic selection for high and low alcohol consumption in a limited-acces paradigm. Alcoholism: Clinical and Experimental Research, 25, 1613-620. doi: 10.1111/j.1530-0277.2001.tb02168.x.

Liu, C., Showalter, J. y Grigson, P. (2009). Ethanol-induced conditioned taste avoidance: reward or aversion?. Alcoholism: Clinical and Experimental Research, 33 (3), 522-530. doi: 10.1111/j.1530-0277.2008.00865.x.

Lu, L., Shepard, J. D., Hall, F. S. y Shaham Y. (2003). Effect of environmental stressors on opiate and psychoestimulant reinforcement, reinstatement and discrimination en rats: a review. Neuroscience \& Biobehavioural Reviews, 27, 457-491. doi: 10.1016/S0149-7634(03)00073-3.

McDevitt-Murphy, M. E., Williams, J. L., Bracken, K. L., Fields, J.A. Monahan, C. J. y Murphy, J. G. (2010). PTSD symptoms, hazardous drinking, and health functioning among U.S. OEF and OIF veterans presenting to primary care. Journal of Traumatic Stress, 23(1), 108-111. doi: $10.1002 /$ jts.20482.

Melis, M., Diana, M., Enrico, P., Marinelli, M. y Brodie, M. (2009). Ethanol and acetaldehyde action on central dopamine systems: mechanisms, modulation, and relationship to stress. Alcohol, 43, 531-539. doi: 10.1016/j.alcohol.2009.05.004

Miczek, K., Yap, J., y Covington, H. (2008). Social stress, therapeutics and drug abuse: Preclinical models of escalated and depressed intake. Pharmacology \& Therapeutics, 120, 102-128. doi: 10.1016/j.pharmthera.2008.07.006.

Pani, L., Pordella, A. y Gessa, G. L. (2000). The role of stress in the pathophysiology of the dopaminergic system. Molecular Psychiatry, 5, 14 21

Pautassi, R. M., Camarini, R., Quadros, I. M., Miczek, K. A. e Israel Y. (2010). Genetic and Environmental Influences on Ethanol Consumption: Perspectives From Preclinical Research. Alcoholism: Clinical and Experimental Research, 34(6), 976-987. doi: 10.1111/j.15300277.2010.01172.x.

Pautassi, R. M., Nizhnikov, M. E. y Spear, N. E. (2009). Assessing appetitive, aversive, and negative ethanol-mediated reinforcement. Neurocience and Biobehavioral Reviews, 33, 953-974. doi: 10.1016/i.neubiorev.2009.03.008.

Philpot, R. M., Badanich, K. A. y Kirstein, C. L. (2003). Place conditioning: Age-related changes in the rewarding and aversive effects of alcohol. 
Alcoholism clinical and experimental research, 27, 593-599. doi: 10.1111/j.1530-0277.2003.tb04395.x.

Quertemont, E., Escarabajal, M. D. y De Witte, P. (2003). Role of catalse in ethanol-induced conditioned taste aversion: a study with 3-amino-1,2,4triazole. Drug and Alcohol Dependence, 70, 77-83. doi: 10.1016/S03768716(02)00341-1.

Roma, P., Davis, C. M., Kohut, S. J., Huntsberry, M. E. y Riley, A. L. (2008). Early maternal separation and sex differences in the aversive effects of amphetamine in adults rats. Physiology of Behavior, 93, 897-904. doi: 10.1016/j.physbeh.2007.12.007.

Roma, P., Rinker, J., Serafine, K., Chen, S., Barr, C., Cheng, K., Rice, K. C. y Riley, A. L. (2008). Genetic and early environmental contributions to alcohol's aversive and physiological effects. Pharmacology, Biochemistry \& Behavior, 91, 134-139. doi: 10.1016/j.pbb.2008.06.022.

Sakusic, A., Avdibegovic, E., Zoricic, Z., Pavlovic, S., Gaspar, V. y Delic, A. (2010). Relationship between anger, alcoholism and symptoms of posttraumatic stress disorders in war veterans. Medical Archives, 64(6), 354-358.

Samson, H. (1986). Initiation of ethanol reinforcement using a sucrosesustitution procedure in food- and water-stated rats. Alcoholism: Clinical and Experimental Research, 10, 436-442.

Sanchis-Segura, C. y Spanagel, R. (2006). Behavioural assessment of drug reinforcement and addictive features in rodents: an overview. Addiction Biology, 11, 2-38. doi: 10.1111/j.1369-1600.2006.00012.x.

See, R. E. y Waters, R. P. (2011). Pharmacologically-induced stress: a crossspecies probe for translational research in drug addiction and relapse. American Journal of Traslational Research, 3(1), 81-89.

Shina, R. (2005). Stress and drug abuse. En T. Steckler, N. Kalin y J. Reul (Eds.), Handbook of stress and the brain (pp. 333-356). Amsterdam: Elsevier.

Shoaib, M., y Almeida, O. (1996). Absence of tolerance to the aversive stimulus properties of ethanol following oral ethanol selfadministration. Alcohol, 13(2), 175-180. doi: 10.1016/07418329(95)02039-X.

Song, M., Wang, X. Y., Zhao, M., Wang, X.-Y., Zhai, H. F., y Lu, L. (2007). Role of stress in acquisition of alcohol-conditioned place preference in adolescent and adult mice. Alcoholism: clinical and experimental research, 31(12), 2001-2005. doi: 10.1111/j.1530-0277.2007.00522.x.

Sorge, R. E., Fudge, M. A. y Parker, L. A. (2002). Effect of sodium deprivation on morphine- and lithium-induced conditioned salt- avoidance and taste reactivity. Psychopharmacology, 160, 84-91. doi: 10.1007/s00213-001-0956-3.

Spear, L. P. y Varlinskaya, E. I. (2010). Sensitivity to ethanol and other hedonic stimuli in an animal model of adolescence: implications for prevention science?. Developmental Psychobiology, 52, 236-43. doi: 10.1002/dev.20457.

Sperling, R. E., Gomes, S. M., Sypek, E. I., Carey, A. N. y McLaughlin, J. P. (2010). Endongenous kappa-opioid mediation of stress-induced potentiation of ethanol-conditioned place preference and selfadministration. Psychopharmacology, 210, 199-209. doi: 10.1007/s00213010-1844-5.

Steckler, T. (2005). The neuropsychology of stress. En T. Steckler, N. H. Kalin, y J. M. Reul (Eds.), Handbook os stress and the Brain (pp. 25-42). Amsterdam: Elsevier.

Tzschentke, T. (1998). Measuring the reward with the conditioned place preference paradigm: a comprehensive review of drigs effects, recent progress and new issues. Progress in Neurobiology, 56, 613- 672. doi: 10.1016/S0301-0082(98)00060-4.

Vengeliene, V., Siegmund, S., Singer, M., Sinclair, J., Li, T. y Spanagel, R. (2003). A comparative study on alcohol-preferring rat lines: effects of deprivation and stress phases on voluntary alcohol intake. Alcoholism: Clinical and Experimental Research, 27, 1048-1054. doi: 10.1097/01.ALC.0000075829.81211.0C.

Vetter-O’Hagen, C., Varlinskaya, E. y Spear, L. (2009). Sex differences in ethanol intake and sensitivity to aversive effects during adolescence and adulhood. Alcohol \& Alcoholism, 44(6), 547-554. doi: $10.1093 /$ alcalc/agp048.

Wilson, M., Burghardt, P., Wilkinson, M. y Primeaux, S. (2004). Anxiolytic effects of diazepam and ethanol in two behavioral models: comparison of males and females. Pharmacology, Biochemistry \& Behavior, 78, 445-448. doi: 10.1016/j.pbb.2004.04.017.

Yap, J. J. y Miczek, K. A. (2008). Stress and rodent models of VTAaccumbens-PFC-amygdala circuit. Drug Discovery Today: Disease Models, 5(4), 259-270. doi: 10.1016/j.ddmod.2009.03.010.

Zimmermann, U. S., Blomeyer, D., Laucht, M. y Mann, K. F. (2007). How gene-stress-behavior interactions can promote adolescent alcohol use: the roles of predrinking allostatic load and childhood behavior disorders. Pharmacology, Biochemistry \& Behavior, 86, 246-262. doi: 10.1016/j.pbb.2006.09.024.

(Articulo recibido: 19-06-2012; revisado: 14-10-2012; aceptado: 14-10-2012) 